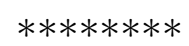

\title{
EFFECTS OF DISORDER IN A DILUTE BOSE GAS
}

\author{
S. Giorgini, L. Pitaevskii* and S. Stringari \\ Dipartimento di Fisica, Universitá di Trento, I-38050 Povo, Italy
}

\begin{abstract}
We discuss the effects of a weak random external potential on the properties of the dilute Bose gas at zero temperature. The results recently obtained by Huang and Meng for the depletion of the condensate and of the superfluid density are recovered. Results for the shift of the velocity of sound as well as for its damping due to collisions with the external field are presented. The damping of phonons is calculated also for dense superfluids.
\end{abstract}

Preprint UTF 253, December 1993 
* Permanent address: Kapitza Institute for Physical Problems, ul. Kosygina 2, 117334 Moscow. 


\section{INTRODUCTION}

The role of disorder in Bose systems has been the object of several theoretical works in the last years ${ }^{1-7}$. In this context an important issue is to understand the effects of disorder on the behaviour of the dilute Bose gas (DBG) where one expects to obtain analytic results at least for weak disorder. This problem was recently considered by the authors of Ref. [3] who investigated the consequences of a random external potential on the depletion of the condensate and of the superfluid component of the system. A striking result emerging from this analysis is that disorder is more active in reducing superfluidity than in depleting the condensate.

The main purpose of the present work is to investigate the consequences of disorder on the propagation of phonons. In particular we study the shift of the sound velocity as well as its damping generated by scattering with the random external potential.

Our formalism is based on dispersive quantum hydrodynamics at zero temperature. This formalism not only accounts in a natural way for the behaviour of the dilute Bose gas, but can be employed, in the hydrodynamic regime, to predict important properties of dense superfluids such as liquid ${ }^{4} \mathrm{He}$.

The paper is organized as follows: in Section 2 we develop the formalism of quantum hydrodynamics in the presence of random external potentials. In Sect. 3 we recover, in the limit of weak disorder, the results of ref.[3] for the depletion of the condensate and of the superfluid density. In Sect.4 we explore the consequences of disorder on the dynamic structure factor. In particular we calculate the change in the velocity of sound and the damping of phonons induced by disorder. The expression for the damping is also extended to the case of strongly interacting superfluids. 


\section{FORMALISM OF QUANTUM HYDRODYNAMICS.}

Let us consider a uniform superfluid system at $T=0$. Our approach to the problem starts from a macroscopic description: we write the energy functional of the system in terms of the superfluid velocity, $\mathbf{v}_{s}$, and the variation of the density with respect to the equilibrium value, $\rho^{\prime}=\rho-\rho_{0}$. We find ${ }^{8}$

$$
\begin{aligned}
E=\frac{\rho_{0}}{2} \int d^{3} r v_{s}^{2}(\mathbf{r})+ & \frac{1}{2} \int d^{3} r \int d^{3} r^{\prime} \chi^{-1}\left(\left|\mathbf{r}-\mathbf{r}^{\prime}\right|\right) \rho^{\prime}(\mathbf{r}) \rho^{\prime}\left(\mathbf{r}^{\prime}\right) \\
& +\frac{1}{2} \int d^{3} r \rho^{\prime}(\mathbf{r}) v_{s}^{2}(\mathbf{r})
\end{aligned}
$$

The superfluid behaviour of the system fixes the irrotational law for the velocity field: $\mathbf{v}_{s}=\nabla \varphi$. In terms of the Fourier components of the density and of the velocity potential, the energy functional can be written as the sum of a quadratic and a cubic term in the fluctuations $\varphi_{\mathbf{k}}$ and $\rho_{\mathbf{k}}^{\prime}: E=H_{0}+H_{1}$, where

$$
H_{0}=\frac{\rho_{0}}{2} \sum_{\mathbf{k}} k^{2}\left|\varphi_{\mathbf{k}}\right|^{2}+\frac{1}{2} \sum_{\mathbf{k}} \chi^{-1}(k)\left|\rho_{\mathbf{k}}^{\prime}\right|^{2}
$$

and

$$
H_{1}=\frac{1}{2 \sqrt{V}} \sum_{\mathbf{k}, \mathbf{k}^{\prime}} \mathbf{k} \cdot \mathbf{k}^{\prime} \varphi_{\mathbf{k}} \rho_{\mathbf{k}^{\prime}-\mathbf{k}}^{\prime} \varphi_{-\mathbf{k}^{\prime}}
$$

The quantity $\chi^{-1}(k)$ is the Fourier transform of $\chi^{-1}(r)$. Terms of third and higher order in $\rho^{\prime}$ have not been included in eqs. (1), (3) since in the DBG limit they do not contribute to the leading order. Applicability of eq. (1) is limited to the region of low temperatures where the whole system is superfluid.

The quadratic functional of equation (2) gives the expression for the energy in the framework of the "harmonic dispersive hydrodynamics", where the compressibility of the system, given by $\chi(k)$, is momentum dependent. In the long wavelength limit the hydrodynamic hamiltonian (2) coincides with the usual phonon hamiltonian with the velocity of sound given by $c=\left(\rho_{0} / \chi(0)\right)^{1 / 2}$. 
Let us neglect for the moment the anharmonic term $H_{1}$ and consider only the harmonic hamiltonian $H_{0}$. By quantizing the fields $\varphi$ and $\rho^{\prime}$ we can cast the hamiltonian into the diagonal form

$$
\hat{H}_{0}=\sum_{\mathbf{k}} \omega_{k} \hat{c}_{\mathbf{k}}^{\dagger} \hat{c}_{\mathbf{k}}
$$

where $\hat{c}_{\mathbf{k}}^{\dagger}\left(\hat{c}_{\mathbf{k}}\right)$ are the creation (annihilation) operators relative to an elementary excitation carrying impulse $\mathbf{k}$ and we have ignored the constant term due to the zero point motion. Equation (4) shows that the system can be described as a gas of non-interacting elementary excitations with energies

$$
\omega_{k}=\sqrt{\frac{\rho_{0} k^{2}}{\chi(k)}} .
$$

The quasiparticle operators $\hat{c}_{\mathbf{k}}^{\dagger}, \hat{c}_{\mathbf{k}}$ are related to the operators $\hat{\varphi}_{\mathbf{k}}$ and $\hat{\rho}_{\mathbf{k}}^{\prime}$ through the following expressions

$$
\begin{gathered}
\delta \hat{\varphi}_{\mathbf{k}}=\hat{\varphi}_{\mathbf{k}}-\left\langle\hat{\varphi}_{\mathbf{k}}\right\rangle=\left(\frac{\omega_{k}}{2 \rho_{0} k^{2}}\right)^{1 / 2}\left(\hat{c}_{\mathbf{k}}+\hat{c}_{-\mathbf{k}}^{\dagger}\right) \\
\delta \hat{\rho}_{\mathbf{k}}=\hat{\rho}_{\mathbf{k}}^{\prime}-\left\langle\hat{\rho}_{\mathbf{k}}^{\prime}\right\rangle=i\left(\frac{\rho_{0} k^{2}}{2 \omega_{k}}\right)^{1 / 2}\left(\hat{c}_{\mathbf{k}}-\hat{c}_{-\mathbf{k}}^{\dagger}\right)
\end{gathered}
$$

where we have introduced the fluctuation operators $\delta \hat{\varphi}_{\mathbf{k}}$ and $\delta \hat{\rho}_{\mathbf{k}}$ which, in the absence of disorder, coincide with the operators $\hat{\varphi}_{\mathbf{k}}$ and $\hat{\rho}_{\mathbf{k}}^{\prime}$.

In the dilute Bose gas limit the frequencies $\omega_{k}$ are given by the most famous Bogoliubov spectrum

$$
\omega_{k}=\frac{k}{2 m}\left(k^{2}+4 \rho_{0} v_{0}\right)^{1 / 2}
$$

and the compressibility $\chi(k)$ takes the form

$$
\chi(k)=\frac{4 \rho_{0} m^{2}}{k^{2}+4 \rho_{0} v_{0}} .
$$


In terms of the scattering length $a$ the interparticle potential $v_{0}$ is written as: $v_{0}=4 \pi a / m$.

Let us now add a perturbative field in the form of a random external potential $U(\mathbf{r})$ coupled to the density through the interaction hamiltonian

$$
\hat{V}=\frac{1}{\sqrt{V}} \sum_{\mathbf{k}} U_{\mathbf{k}} \hat{\rho}_{-\mathbf{k}}^{\prime}
$$

In eq. (9) $U_{\mathbf{k}}$ is the Fourier transform of the external potential. In order to investigate in a simple way how the random potential affects the behaviour of the system, we will often make the white noise assumption in which the external potential is described by a single parameter $R_{0}[3]$ :

$$
\frac{1}{V}\left\langle\left|U_{\mathbf{k}}\right|^{2}\right\rangle=\frac{R_{0}}{m^{2}}
$$

and where the average is assumed to be of Gaussian type. In eq. (10) $m$ is the mass of the particles and the dimension of $R_{0}$ is consequently (energy $)^{2} \times\left(\right.$ length) ${ }^{3}$.

In the presence of weak disorder the expectation value of the density operator on the ground state can be evaluated by means of linear response theory:

$$
\left\langle\hat{\rho}_{\mathbf{k}}^{\prime}\right\rangle=-\frac{1}{\sqrt{V}} U_{\mathbf{k}} \chi(k)
$$

whereas the expectation value of the velocity potential operator is not affected by the external static field: $\left\langle\hat{\varphi}_{\mathbf{k}}\right\rangle=0$. By writing both the hamiltonians $\hat{H}_{0}$ and $\hat{V}$ in terms of the operators $\hat{c}_{\mathbf{k}}, \hat{c}_{\mathbf{k}}^{\dagger}$ we get the result

$$
\hat{H}=-\frac{\rho_{0}}{2} \sum_{\mathbf{k}} \frac{1}{V}\left\langle\left|U_{\mathbf{k}}\right|^{2}\right\rangle \frac{k^{2}}{\omega_{k}^{2}}+\sum_{\mathbf{k}} \omega_{k} \hat{c}_{\mathbf{k}}^{\dagger} \hat{c}_{\mathbf{k}}
$$

for the harmonic hamiltonian $\hat{H}=\hat{H}_{0}+\hat{V}$. The first term in (12) gives the correction to the ground state energy due to the external field. This quantity can be calculated 
also beyond the dilute Bose gas approximation. In fact by applying linear reponse theory (eq.(11)) to the external field (9) one finds the general result

$$
\delta E=-\frac{1}{2} \sum_{\mathbf{k}} \frac{1}{V}\left\langle\left|U_{\mathbf{k}}\right|^{2}\right\rangle \chi(k)
$$

holding also for strongly interacting systems. Equation (13) reduces to the first term in the right hand side of eq.(12) in the Bogoliubov limit where eq.(5) holds. It is important to notice that if one makes the white noise assumption (10), the correction to the ground state energy (13) contains an ultraviolet divergence since

$\chi(k)$ behaves as $k^{-2}$ for large $k$. A more physical choice for $\frac{1}{V}\left\langle\left|U_{\mathbf{k}}\right|^{2}\right\rangle$ would not yield such a divergence.

From result (12) it turns out that, without including interaction terms among the quasiparticles, described by the anharmonic term $H_{1}$, the energies of the elementary excitations are not affected by the random external field; in particular the velocity of sound is still given by the Bogoliubov value $c=\left(\rho_{0} v_{0}\right)^{1 / 2} / m$. In Section 4 we will calculate the first correction to the velocity of sound as well as its damping due to the random external potential.

\section{SUPERFLUID DENSITY AND DEPLETION OF THE CONDEN-}

\section{SATE}

The current density associated with the one-fluid hamiltonian (1) is given by

$$
\hat{\mathbf{j}}(\mathbf{r})=\frac{\partial E}{\partial \mathbf{v}_{s}}=\rho_{0} \hat{\mathbf{v}}_{s}(\mathbf{r})+\hat{\rho}^{\prime}(\mathbf{r}) \hat{\mathbf{v}}_{s}(\mathbf{r})
$$

where we have separeted the first and the second order terms. By taking the Fourier transform of the current operator we obtain

$$
\hat{\mathbf{j}}_{\mathbf{q}}=i \mathbf{q} \rho_{0} \hat{\varphi}_{\mathbf{q}}+\frac{1}{\sqrt{V}} \sum_{\mathbf{k}} i \mathbf{k} \hat{\varphi}_{\mathbf{k}} \hat{\rho}_{\mathbf{q}-\mathbf{k}}^{\prime}
$$


The first term is longitudinal, whereas the second one contains both a longitudinal as well as a transverse component. By inserting in eq. (15) the expressions for

the velocity potential and density (6) in terms of the quasiparticle operators $\hat{c}_{\mathbf{k}}^{\dagger}, \hat{c}_{\mathbf{k}}$, one can distinguish in a natural way between different contributions to the current operator. The first term in (15) is coupled to single elementary excitations: we call this term the one-phonon contribution to the current. The second term can be either the product of one quasiparticle operator and of the external field (hereafter referred to as disorder contribution) or the product of two quasiparticle operators (two-phonon contribution).

Let us choose $\mathbf{q}$ in the $z$ direction: $\mathbf{q}=(0,0, q)$ and let us consider the tranverse component of the current operator $\hat{j}_{\mathbf{q}}^{x}$. In terms of the elementary excitation operators it is written as

$$
\hat{j}_{\mathbf{q}}^{x}=\hat{j}_{\mathbf{q}}^{x(1)}+\hat{j}_{\mathbf{q}}^{x(2)}=-\frac{1}{V} \sum_{\mathbf{k}} i k_{x}\left(\frac{\rho_{0} \omega_{k}}{2}\right)^{1 / 2} \frac{U_{\mathbf{q}-\mathbf{k}}|\mathbf{q}-\mathbf{k}|^{2}}{\omega_{\mathbf{q}-\mathbf{k}}^{2} k}\left(\hat{c}_{\mathbf{k}}+\hat{c}_{-\mathbf{k}}^{\dagger}\right)+\hat{j}_{\mathbf{q}}^{x(2)}
$$

where the operator $\hat{j}_{\mathbf{q}}^{x(2)}$ arises from two-phonon effects and does not depend upon the external field.

There is an important point to stress here. The transverse current operator (arising from the second term in the r.h.s. of eq. (15)) is fixed by the anharmonic term in the hamiltonian. This implies that, for a calculation of the corresponding matrix elements to the lowest order, we can safely use the eigenstates and eigenvalues of the harmonic hamiltonian $\hat{H}_{0}$. The situation is different in the longitudinal channel, since the longitudinal component of the current operator (15) contains a term, the one-phonon contribution, which is zeroth order in the anharmonic perturbation. In the next Section we will see that the anharmonic term $\hat{H}_{1}$ has to be explicitly included in the hamiltonian in order to study the longitudinal channel in the proper way. 
The normal component of the fluid is obtained by considering the static transverse current-current response which, at $T=0$, can be written as

$$
\chi^{T}(q)=\sum_{n} \frac{\left|\left\langle 0\left|\hat{j}_{\mathbf{q}}^{x}\right| n\right\rangle\right|^{2}}{\omega_{n}}+\frac{\left|\left\langle 0\left|\hat{j}_{-\mathbf{q}}^{x}\right| n\right\rangle\right|^{2}}{\omega_{n}} .
$$

The normal density is then obtained from the long wavelength limit of equation $(17)^{9}$

$$
\rho_{n}=\lim _{q \rightarrow 0} \chi^{T}(q)
$$

while the superfluid density is defined as $\rho_{s}=\rho_{0}-\rho_{n}$. In eq. (17) $|0\rangle$ is the ground state and the sum is performed over a complete set of excited states with energies $\omega_{n}$. If we now insert the expression (16) for the current operator into eq. (17) we can distinguish the contribution to the response function arising from two-phonon effects from the one fixed by disorder. The former can be shown to vanish in the long wavelength limit as $q^{2}$. The latter is easily obtained by a direct calculation of the matrix elements using the diagonal hamiltonian (4). The final result for the normal density is given by

$$
\rho_{n}=\rho_{0} \frac{1}{V} \sum_{\mathbf{k}} k_{x}^{2} \frac{k^{2}}{\omega_{k}^{4}} \frac{1}{V}\left\langle\left|U_{\mathbf{k}}\right|^{2}\right\rangle .
$$

The fact that the random external potential gives rise to a normal component in the fluid can be also regarded as the consequence of the fact that translational invariance is broken by the external field and therefore the current is not conserved ${ }^{10}$.

Result (19) needs some comments. First of all eq. (19) shows that the normal component induced by the random external potential is not fixed just by the long wavelength properties of the system. In order to assure the convergence of the integral in (19) the behaviour of the elementary excitation spectrum at high momenta is important. A second point concerns the validity of the one-fluid hamiltonian (1) when a normal component is present in the fluid. Result (19) can be 
interpreted as the first term of the perturbative expansion of the normal density in the external field $U$. It is finally worth noticing that in the case of an ideal Bose gas $\left(\omega_{k}=k^{2} / 2 m\right)$ the integral in eq. (19) diverges at small $k$ 's revealing the instability of the ideal Bose gas in the presence of disorder.

In the DBG limit the integral in (19) can be calculated analytically by inserting the Bogoliubov value ( 7 ) for the energies $\omega_{k}$ and using the white noise approximation for $\frac{1}{V}\left\langle\left|U_{\mathbf{k}}\right|^{2}\right\rangle$. One gets

$$
\rho_{n}=\frac{m^{3}}{6 \pi^{3 / 2}} R_{0}\left(\frac{n}{a}\right)^{1 / 2}
$$

where $n$ is the particle density. The applicability of result (20) is subject to the condition of weak disorder $\rho_{n}<<\rho_{0}$ or, equivalently,

$$
\frac{m^{2} R_{0}}{\sqrt{n a}}<<1
$$

In $2 \mathrm{D}$ one finds

$$
\rho_{n}=\frac{m^{3}}{8 \pi^{2}} R_{0} \frac{1}{a}
$$

Both eqs.(20) and (22) are in agreement with the results of Refs. [3,11].

We now calculate the depletion of the condensate due to the random potential. To this purpose let us write the macroscopic component of the particle field operator $\hat{\Psi}(\mathbf{r})$, in terms of density and phase fluctuations. The macroscopic component of the particle operator is formally given by

$$
\hat{\Psi}_{M}(\mathbf{r})=\sqrt{\hat{n}_{0}} e^{i \hat{\Phi}(\mathbf{r})}
$$

where $\hat{n}_{0}$ is the operator of the number of particles in the condensate and $\hat{\Phi}(\mathbf{r})$ is the phase operator related to the velocity potential by the usual relation $\hat{\varphi}(\mathbf{r})=\hat{\Phi}(\mathbf{r}) / m$. In the DBG limit, where $n_{0} \simeq n=\rho_{0} / m$, the following expansion is valid up to first order in the density and phase fluctuations

$$
\hat{\Psi}_{M}(\mathbf{r})=\sqrt{n}\left(1+\frac{1}{2 n m} \hat{\rho}^{\prime}(\mathbf{r})+i \hat{\Phi}(\mathbf{r})\right)
$$


By taking the Fourier transform of eq. (24) one finds

$$
\delta \hat{a}_{\mathbf{q}}=\hat{a}_{\mathbf{q}}-\left\langle\hat{a}_{\mathbf{q}}\right\rangle=i \sqrt{n} \delta \hat{\Phi}_{\mathbf{q}}+\frac{1}{2 \sqrt{n} m} \delta \hat{\rho}_{\mathbf{q}}
$$

with $\left\langle\hat{a}_{\mathbf{q}}\right\rangle=\sqrt{n} \delta_{\mathbf{q}, 0}+1 /(2 \sqrt{n} m)\left\langle\hat{\rho}_{\mathbf{q}}^{\prime}\right\rangle$ (we have used $\left\langle\hat{\Phi}_{\mathbf{q}}\right\rangle=0$ ). The momentum distribution is thus given by

$$
n(q)=\left\langle\delta \hat{a}_{\mathbf{q}}^{\dagger} \delta \hat{a}_{\mathbf{q}}\right\rangle+\left|\left\langle\hat{a}_{\mathbf{q}}\right\rangle\right|^{2}=\frac{\rho_{0}}{m}\left\langle\delta \hat{\Phi}_{-\mathbf{q}} \delta \hat{\Phi}_{\mathbf{q}}\right\rangle+\frac{1}{4 m \rho_{0}}\left\langle\delta \hat{\rho}_{-\mathbf{q}} \delta \hat{\rho}_{\mathbf{q}}\right\rangle-\frac{1}{2}+\frac{1}{4 m \rho_{0}}\left|\left\langle\hat{\rho}_{\mathbf{q}}^{\prime}\right\rangle\right|^{2}
$$

The first term in the right hand side of equation (26) represents the contribution to the momentum distribution given by the phase fluctuations. In the long wavelength limit this term gives the main contribution and is responsible for the well known $1 / q$ divergence of $n(q)^{12}$. In the region of higher momenta also the density fluctuations $\left\langle\delta \hat{\rho}_{-\mathbf{q}} \delta \hat{\rho}_{\mathbf{q}}\right\rangle$ become important. The constant term $-1 / 2$ arises from the commutation relation between density and phase operators. It is worth noticing that the random potential explicitly affects the result for the momentum distribution because the expectation value of the particle operator $\hat{a}_{\mathbf{q}}$ on the ground state is no longer zero for $\mathbf{q} \neq 0$ :

$$
\left\langle\hat{a}_{\mathbf{q}}\right\rangle=\frac{1}{2 \sqrt{n} m}\left\langle\hat{\rho}_{\mathbf{q}}^{\prime}\right\rangle=-\frac{1}{2 \sqrt{n} m} \frac{1}{\sqrt{V}} U_{\mathbf{q}} \chi(q)
$$

By using the Bogoliubov results for the density and phase fluctuations (corrections to these quantities due to disorder give rise to higher order effects) we finally obtain the result

$$
n(q)=\frac{1}{2 \omega_{q}}\left(\frac{q^{2}}{2 m}+n v_{0}-\omega_{q}\right)+\frac{n}{4} \frac{1}{V}\left\langle\left|U_{\mathbf{q}}\right|^{2}\right\rangle \frac{q^{4}}{\omega_{q}^{4}}
$$

where the first term coincides with the prediction of the Bogoliubov model. The depletion is obtained by integrating result (28) and we find the usual Bogoliubov 
value with a correction proportional to $\rho_{n}$ :

$$
\Delta n=n-n_{0}=\frac{1}{V} \sum_{\mathbf{q} \neq 0} n(q)=\frac{8}{3 \pi^{1 / 2}}(n a)^{3 / 2}+\frac{3}{4 m} \rho_{n}
$$

Result (29) is in agreement with the findings of Ref. [3]. In particular it shows that the relative depletion of the condensate due to disorder is a factor $3 / 4$ smaller than the corresponding reduction of the superfluid density. This result holds for

any choice of the average $\frac{1}{V}\left\langle\left|U_{\mathbf{k}}\right|^{2}\right\rangle$ since the same integral over momenta is involved in the calculation of both $\rho_{n}$ and $\Delta n$ (see eqs.(19) and (28)). We stress however that eq. (29) holds only in the presence of weak disorder. It cannot be easily extrapolated to the large external fields.

\section{VELOCITY AND DAMPING OF SOUND}

In the present Section we discuss the effect of disorder on the dispersion of the phonon mode at $T=0$. To this purpose we first calculate the compressibility of the system, $\chi(0)$, defined by the relation

$$
\chi^{-1}(0)=\frac{\partial^{2}}{\partial \rho^{2}} \frac{E}{V}
$$

where $E$ is the ground state energy of the system. The contribution to $E$ due to disorder is given by eq.(13) or equivalently, in the Bogoliubov limit, by the first term in the r.h.s. of eq. (12). After taking the second derivative with respect to the density we find the result

$$
\chi^{-1}(0)=\chi_{0}^{-1}(0)\left(1+\sum_{\mathbf{k}} \frac{1}{V}\left\langle\left|U_{\mathbf{k}}\right|^{2}\right\rangle \frac{k^{8}}{4 \omega_{k}^{6}}\right)
$$

where $\chi_{0}(0)$ is the compressibility of the DBG. By making the white noise assumption (10) it is possible to relate result (31) to the normal density $\rho_{n}$ given by eq.(19). We find

$$
\chi^{-1}(0)=\chi_{0}^{-1}(0)\left(1+\frac{9}{4} \frac{\rho_{n}}{\rho_{0}}\right)
$$


It is worth noticing that, differently from the correction to the total energy as well as to the first derivative $\partial E / \partial \rho$ (proportional to the chemical potential), the contribution of disorder to the compressibility is well defined also within the white noise assumption. This is due to the fact that the quantity $\partial^{2} \chi(k) / \partial \rho^{2}$, differently from $\chi$ and $\partial \chi(k) / \partial \rho$ decreases as $k^{-4}$ when $k \rightarrow \infty$ and the corresponding integral is well behaved for large $k$.

We are now ready to calculate the velocity of sound. Using the relation

$$
c^{2}=\frac{\rho_{s}}{\chi(0)}
$$

typical of superfluids and eqs. (20) and (32) for $\rho_{n}$ and $\chi(0)$ we obtain the result

$$
c^{2}=c_{0}^{2}\left(1+\frac{5}{4} \frac{\rho_{n}}{\rho_{0}}\right)
$$

holding within the white noise assumption. Equation (34) shows that the velocity of sound increases with disorder and that consequently the phonon peak in the dynamic structure function

$$
S(q, \omega)=\int_{-\infty}^{+\infty} d t e^{i \omega t}\left\langle\left[\delta \hat{\rho}_{-\mathbf{q}}(t), \delta \hat{\rho}_{\mathbf{q}}(0)\right]\right\rangle
$$

is shifted to the right according to the law

$$
S_{\text {phonon }}(q, \omega)=\frac{\rho_{s} q}{2 c} \delta(\omega-c q)
$$

The normalization factor $\rho_{s} q / 2 c$ ensures that the phonon exhausts the compressibility sum rule

$$
\chi(0)=2 \lim _{q \rightarrow 0} \int \frac{1}{\omega} S(q, \omega) d \omega
$$

consistently with relation (33) for the sound velocity. 
In the second part of the section we calculate the damping of phonons due to collisions with the external potential. To this purpose we calculate directly the propagator defined by the time ordered product

$$
D_{11}(q, t)=-i\left\langle T\left(\hat{\rho}_{\mathbf{q}}^{\prime}(t) \hat{\rho}_{-\mathbf{q}}^{\prime}(0)\right)\right\rangle=-i\left\langle\left(\theta(t) \hat{\rho}_{\mathbf{q}}^{\prime}(t) \hat{\rho}_{-\mathbf{q}}^{\prime}(0)+\theta(-t) \hat{\rho}_{-\mathbf{q}}^{\prime}(0) \hat{\rho}_{\mathbf{q}}^{\prime}(t)\right)\right\rangle
$$

in terms of which the dynamic structure function is written as $S(q, \omega)=-\frac{1}{\pi} \operatorname{Im} D_{11}(q, \omega)$.

As anticipated in Section 2 in order to calculate to the proper order the effects of the external random potential in the longitudinal channel it is essential to include the anharmonic term (3) in the hamiltonian of the system.

We calculate the imaginary part of the propagator $D_{11}(\mathbf{q}, \omega)$ perturbatively in the interaction hamiltonian

$$
\hat{H}_{i n t}=\hat{H}_{1}+\hat{V}=\frac{1}{2 \sqrt{V}} \sum_{\mathbf{k}, \mathbf{k}^{\prime}} \mathbf{k} \cdot \mathbf{k}^{\prime} \hat{\varphi}_{\mathbf{k}} \hat{\rho}_{\mathbf{k}^{\prime}-\mathbf{k}}^{\prime} \hat{\varphi}_{-\mathbf{k}^{\prime}}+\frac{1}{\sqrt{V}} \sum_{\mathbf{k}} U_{\mathbf{k}} \hat{\rho}_{-\mathbf{k}}^{\prime}
$$

up to terms of second order in the random potential $\hat{V}$. The calculation is straightforward and the details are given in the Appendix. The relevant contribution to the imaginary part of the propagator arises from the diagram of Figure 1. By adding this contribution to the phonon peak (36) we find, for small $q$, the result

$$
S(q, \omega)=\frac{\rho_{s} q}{2 c} \delta(\omega-c q)+\frac{\omega^{2} q^{2}}{\left(\omega^{2}-c^{2} q^{2}\right)^{2}} \frac{\rho_{0}}{2 V} \sum_{\mathbf{k}} \frac{1}{V}\left\langle\left|U_{\mathbf{k}}\right|^{2}\right\rangle k_{z}^{2} \frac{k^{2}}{\omega_{k}^{3}} \delta\left(\omega-\omega_{k}\right)
$$

where the second term holds for $\omega$ not too close to $c q$ : $|\omega-c q|>c q$. In the hydrodynamic limit $(q \rightarrow 0, \omega \rightarrow 0)$ result $(40)$ becomes

$$
S(q, \omega)=\frac{\rho_{s} q}{2 c} \delta(\omega-c q)+\frac{q^{2} \omega^{5}}{\left(\omega^{2}-c^{2} q^{2}\right)^{2}} \frac{\rho_{0}}{12 \pi^{2} c^{7}} \frac{1}{V}\left\langle\left|U_{\mathbf{k}}\right|^{2}\right\rangle_{|\mathbf{k}|=\omega / \mathbf{c}}
$$

Eq. (41) corresponds, in the limit of weak disorder (see eq.(21)), to the first two terms of the series of the most divergent diagrams characterizing the behaviour of 
the propagator $D_{11}$, near the pole. The sum of this series yields the following result for $S(q, \omega)$ near the pole

$$
S(q, \omega)=\frac{\rho_{s} q^{2}}{\pi} \frac{2 c q \Gamma(q)}{\left(\omega^{2}-c^{2} q^{2}\right)^{2}+4 c^{2} q^{2} \Gamma^{2}(q)}
$$

with the width $\Gamma(q)$ given by

$$
\Gamma(q)=\frac{1}{24 \pi} \frac{1}{c^{3}} q^{4} \frac{1}{V}\left\langle\left|U_{\mathbf{q}}\right|^{2}\right\rangle
$$

Eqs. (42), (43) explicitly show that the phonon peak is broadened by the presence of disorder.

It is not difficult to extend results (42), (43) to the case of a strongly interacting Bose superfluid where quantum hydrodynamics is expected to provide the proper description in the hydrodynamic regime. To this purpose we have to add to the anharmonic term (3) a contribution cubic in the density fluctuations yielding, for $\hat{H}_{1}$, the more general expression ${ }^{13}$

$$
\hat{H}_{1}=\frac{1}{2 \sqrt{V}} \sum_{\mathbf{k}, \mathbf{k}^{\prime}} \mathbf{k} \cdot \mathbf{k}^{\prime} \hat{\varphi}_{\mathbf{k}} \hat{\rho}_{\mathbf{k}^{\prime}-\mathbf{k}}^{\prime} \hat{\varphi}_{-\mathbf{k}^{\prime}}+\frac{1}{6 \sqrt{V}}\left(\frac{d}{d \rho} \frac{c^{2}}{\rho}\right) \sum_{\mathbf{k} \mathbf{k}^{\prime}} \hat{\rho}_{\mathbf{k}}^{\prime} \hat{\rho}_{\mathbf{k}^{\prime}-\mathbf{k}}^{\prime} \hat{\rho}_{-\mathbf{k}^{\prime}}^{\prime}
$$

By accounting for the diagrams relevant for the calculation of the damping we get the relevant result (see the Appendix for details)

$$
\Gamma(q)=\frac{1}{24 \pi} \frac{q^{4}}{c^{3}} \frac{1}{V}\left\langle\left|U_{\mathbf{q}}\right|^{2}\right\rangle\left(1+3 \frac{\rho_{0}^{4}}{c^{4}}\left(\frac{d}{d \rho} \frac{c^{2}}{\rho}\right)^{2}\right) .
$$

In superfluid ${ }^{4}$ He the second term in eq. (45) turns out to be of the same order as the first one. Vice-versa in the DBG the derivative with respect to the density of $c^{2} / \rho$ is of higher order in the scattering length and its contribution can be consequently neglected. Notice that the damping mechanism discussed above is dominant at small $q$ compared to the damping $\Gamma_{p h-p h}$ due to anharmonic interactions among 
phonons, which exhibits a $q^{5}$ law (see Ref. [13]). In the dilute Bose gas and using the white noise approximation we find the result

$$
\frac{\Gamma(q)}{\Gamma_{p h-p h}(q)}=\frac{20}{3} \frac{\rho_{n}}{\rho_{0}} \frac{1}{q a}
$$

Starting from result (40) we can finally calculate the low $q$ behaviour of various energy moments of the dynamic structure factor. These moments can be written as the sum of the phonon contribution (first term in eq. (40)) and of the collisional term (second term of eq. (40)). The latter contribution is always proportional to $q^{2}$. For example, by integrating eq.(40) with respect to $\omega$, one finds the following result for the low $q$ expansion of the non energy weigthed sum rule (static structure factor):

$$
S(q)=\int_{0}^{\infty} d \omega S(q, \omega)=\frac{\rho_{s} q}{2 c}+q^{2} \frac{\rho_{0}}{2} \int d \mathbf{k} \frac{1}{(2 \pi)^{3}} \frac{1}{V}\left\langle\left|U_{\mathbf{k}}\right|^{2}\right\rangle \frac{k_{z}^{2} k^{2}}{\omega_{k}^{5}}
$$

By making the white noise approximation for $\frac{1}{V}\left\langle\left|U_{\mathbf{k}}\right|^{2}\right\rangle$, one can relate the second term to the normal density (19):

$$
S(q)=\frac{\rho_{s} q}{2 c}+q^{2} \rho_{n} \frac{m}{12 \pi^{2} n a}
$$

Eq. (48) shows that the static structure factor, at low $q$, is exhausted by the phonon peak. One immediately shows that the phonon peak exhausts also the inverse energy weighted sum-rule fixed by the compressibility of the system (eq. (37)).

For the energy weighted sum rule we find the result

$$
m_{1}=\int_{0}^{\infty} d \omega \omega S(q, \omega)=\rho_{s} \frac{q^{2}}{2}+\rho_{n} \frac{q^{2}}{2}=\rho_{0} \frac{q^{2}}{2}
$$

which coincides, as expected, with the model independent f-sum rule, proportional to the total density of the system. In this case the contribution of the collisional term is crucial in order to satisfy the sum rule also at low $q$. This contribution 
can be always expressed in terms of the normal density, independently of the white noise assumption. It is interesting to remark that a similar behaviour is exhibited by transverse spin excitations (magnons) in antiferromagnets ${ }^{14}$. Also in this case the magnon exhausts only a fraction of the energy weighted sum rule, the remaining part being, in this case, exhausted by multimagnon excitations. The one-magnon contribution to the energy weighted sum rule is proportional to the spin stiffness coefficient which plays the role of the superfluid density. This analogy between disordered bosons and antiferromagnets is due to the fact that in both cases the current is not conserved due to the lack of translational invariance.

The fact that the phonon peak does not exhaust the f-sum rule implies that the Feynman approximation

$$
\omega_{F}(q)=\frac{\rho_{0} q^{2}}{2 S(q)}
$$

for the energy of elementary excitations does not coincide with the phonon dispersion at low $q$. Actually using result (47) for $S(q)$ one finds $\omega_{F}(q) \rightarrow c q \rho_{0} / \rho_{s}$ a value higher than $c q$. It is also worth noticing that the Feynman ratio (50) is affected by the $q^{2}$ correction in $S(q)$. Such a correction is absent in translationally invariant systems.

\section{ACKNOWLEDGMENTS}

We wish to thank K. Huang for useful discussions. L.P. likes to thank the hospitality of the Department of Physics of the University of Trento. 


\section{APPENDIX}

In this appendix we evaluate the imaginary part of the density-density propagator $D_{11}(\mathbf{q}, \omega)$ in the long wavelength limit, to first order in $R_{0}$. We use time dependent perturbation theory with the perturbative field given in equation (39).

We need the free field propagators:

$$
\begin{gathered}
i D_{11}^{0}(q, \omega)=\int_{-\infty}^{+\infty} d t e^{i \omega t}\left\langle T\left(\hat{\rho}_{\mathbf{q}}^{\prime}(t) \hat{\rho}_{-\mathbf{q}}^{\prime}(0)\right)\right\rangle_{0}=i \frac{\rho_{0} q^{2}}{\omega^{2}-\omega_{q}^{2}+i 0} \\
i D_{22}^{0}(q, \omega)=\int_{-\infty}^{+\infty} d t e^{i \omega t}\left\langle T\left(\hat{\varphi}_{\mathbf{q}}(t) \hat{\varphi}_{-\mathbf{q}}(0)\right)\right\rangle_{0}=i \frac{\omega_{q}^{2}}{\rho_{0} q^{2}} \frac{1}{\omega^{2}-\omega_{q}^{2}+i 0} \\
i D_{12}^{0}(q, \omega)=\int_{-\infty}^{+\infty} d t e^{i \omega t}\left\langle T\left(\hat{\rho}_{\mathbf{q}}^{\prime}(t) \hat{\varphi}_{-\mathbf{q}}(0)\right)\right\rangle_{0}=-\frac{\omega}{\omega^{2}-\omega_{q}^{2}+i 0}
\end{gathered}
$$

where the subscript after the brackets $\langle\ldots\rangle_{0}$ means that the expectation value is taken on the ground state relative to the unperturbed hamiltonian $H_{0}$.

The density-density propagator is obtained from the general formula of perturbation theory

$$
i D_{11}(\mathbf{q}, t)=\frac{1}{\langle\hat{S}\rangle}\left\langle T\left(\hat{\rho}_{\mathbf{q}}^{\prime}(t) \hat{\rho}_{-\mathbf{q}}^{\prime}(0) \hat{S}\right)\right\rangle
$$

where the time evolution operator $\hat{S}$ is defined by the series expansion

$$
\hat{S}=\sum_{n=0}^{\infty} \frac{(-i)^{n}}{n !} \int_{-\infty}^{+\infty} d t_{1} \ldots \int_{-\infty}^{+\infty} d t_{n} T\left(\hat{H}_{i n t}\left(t_{1}\right) \ldots \hat{H}_{i n t}\left(t_{n}\right)\right)
$$

It is better to use diagrams to represent the different terms arising from equation (A4) after using Wick's theorem for time ordered products. The anharmonic potential $\hat{H}_{1}$ is represented by a vertex with three lines, whereas to the external random potential corresponds a vertex with just one line.

At finite frequencies the leading correction to the imaginary part of $D_{11}(\mathbf{q}, \omega)$

comes from terms which are fourth order in the interaction hamiltonian $\hat{H}_{\text {int }}$. These 
terms correspond to diagrams containing two vertices relative to the anharmonic potential and two random potential vertices.

It turns out that only one diagram is relevant and this is given in figure 1, where we have represented $D_{11}^{0}$ by a full line, $D_{22}^{0}$ by a dashed line and $D_{12}^{0}$ by a long-dashed line. In fact the external random field is time independent. This means that only the density-density propagator, which has a zero frequency component, can be connected to the random potential vertices. The contribution arising from the diagram in figure 1 to the density-density propagator is given by

$$
\begin{aligned}
& D_{11}(\mathbf{q}, \omega)=D_{11}^{0}(q, \omega)+\frac{\omega^{2}}{\rho_{0}^{2} q^{2}}\left(D_{11}^{0}(q, \omega)\right)^{2} \\
& \times \frac{1}{V} \sum_{\mathbf{k}} \frac{1}{V}\left\langle\left|U_{\mathbf{k}+\mathbf{q}}\right|^{2}\right\rangle k_{z}^{2} D_{22}^{0}(k, \omega)\left(D_{11}^{0}(|\mathbf{k}+\mathbf{q}|, 0)\right)^{2}
\end{aligned}
$$

The square of the free propagator $D_{11}^{0}$ in equation (A6) can be approximated, for $\omega$ not too close to the pole $\left(\left|\omega-\omega_{q}\right|>\omega_{q}\right)$, with the expression

$$
\left(D_{11}^{0}(q, \omega)\right)^{2}=\frac{\rho_{0}^{2} q^{4}}{\left(\omega^{2}-\omega_{q}^{2}\right)^{2}}
$$

Result (40) for the dynamic structure factor follows directly from equations (A6) by using the relation $S(\mathbf{q}, \omega)=-\frac{1}{\pi} \operatorname{Im} D_{11}(\mathbf{q}, \omega)$ and after taking the $q \rightarrow 0$ limit.

The imaginary part of the density-density self energy can be directly evaluated from equation (A6). In the long wavelength limit one gets

$$
-\operatorname{Im} \Sigma_{11}(q, \omega)=\frac{\pi}{2} \frac{\omega^{2}}{\rho_{0} q^{2}} \frac{1}{V} \sum_{\mathbf{k}} \frac{1}{V}\left\langle\left|U_{\mathbf{k}}\right|^{2}\right\rangle k_{z}^{2} \frac{k^{2}}{\omega_{k}^{3}} \delta\left(\omega-\omega_{k}\right)
$$

Result (43) for the damping in the phonon region follows from eq. (A8) by using the relation

$$
\Gamma(q)=\frac{\rho_{0} q}{2 c}\left(-\operatorname{Im} \Sigma_{11}(q, \omega=c q)\right)
$$


In the case of strongly correlated systems the damping of the phonon mode can be obtained in the long wavelength limit by using the anharmonic term (44). In this case another diagram is relevant to the calculation of the damping and it is given in figure 2. The imaginary part of the self energy is given by

$$
-\operatorname{Im} \Sigma_{11}(q, \omega)=\frac{\pi}{2} \frac{1}{\rho_{0}} \frac{1}{V} \sum_{\mathbf{k}} \frac{1}{V}\left\langle\left|U_{\mathbf{k}}\right|^{2}\right\rangle\left(k_{z}^{2} \frac{\omega^{2} k^{2}}{\omega_{k}^{3} q^{2}}+\rho_{0}^{4}\left(\frac{d}{d \rho} \frac{c^{2}}{\rho}\right)^{2} \frac{k^{6}}{\omega_{k}^{5}}\right) \delta\left(\omega-\omega_{k}\right)
$$

and result (45) follows directly from relation (A9).

The present diagrammatic technique could be used for the calculation of higher order corrections in the parameter $R_{0}$. To perform the corresponding averaging one must assume gaussian statistics for the random external field. 


\section{REFERENCES}

${ }^{1}$ M. Ma, B.I. Halperin and P.A. Lee, Phys. Rev. B, 34, 3136 (1986).

${ }^{2}$ M.P.A. Fisher, P.B. Weichman, G. Grinstein and D.S. Fisher, Phys. Rev. B 40, 546 (1989).

${ }^{3}$ K. Huang and H.F. Meng, Phys. Rev. Lett. 69, 644 (1992).

${ }^{4}$ W. Krauth, N. Trivedi and D. Ceperley, Phys. Rev. Lett. 67, 2307 (1991).

${ }^{5}$ M. Makivic, N. Trivedi and S. Ullah, Phys. Rev. Lett. 71, 2307 (1993).

${ }^{6}$ L. Zhang, Phys. Rev. B 47, 14364 (1993).

${ }^{7}$ P. Nisamaneephong, L. Zhang and M. Ma, Phys. Rev. Lett. 71, 3830 (1993).

${ }^{8}$ L.P. Pitaevskii, J. Exp. Theoret. Phys. (U.S.S.R.) 31, 536 (1956).

${ }^{9}$ G. Baym, in Mathematical Methods in Solid State and Superfluid Theory,

R.C.Clark and G.H.Derrick eds. (Oliver and Boyd, Edinburgh, 1969),p.151.

${ }^{10}$ S. Stringari, in Bose Einstein Condensation, A.Griffin, D.Snoke and S.Stringari eds. (Cambridge University Press, in press).

${ }^{11}$ H.F. Meng, Ph. D. thesis, MIT (unpublished).

12 T. Gavoret and Ph. Nozieres, Ann. Phys. (N.Y.) 28,349 (1964).

${ }^{13}$ E.M. Lifshitz and L.P. Pitaevskii, Statistical Physics

(Pergamon,Oxford, 1980), Part 2, pp. 98 and 134.

${ }^{14}$ S. Stringari, (Phys. Rev. B. in press). 


\section{FIGURE CAPTIONS}

Fig.1. The relevant diagram of the fourth order in $\hat{H}_{i n t}$ (eq. (39)). The full line represents $D_{11}^{0}$, the dashed line $D_{22}^{0}$ and the long-dashed line $D_{12}^{0}$.

Fig.2. The other relevant diagram of the fourth order in $\hat{H}_{i n t}=\hat{H}_{1}+\hat{V}$ with $\hat{H}_{1}$ given by eq. (44). The full line represents $D_{11}^{0}$. 